\title{
EXPERIMENTAL INVESTIGATION OF LARGE PLASTIC DEFORMATION AND FRACTURE IN EXPLOSIVELY LOADED OPEN-ENDED STEEL CYLINDERS
}

\author{
G.K. SCHLEYER ${ }^{1} \&$ N.J. RUSHTON ${ }^{2}$ \\ ${ }^{1}$ University of Liverpool, School of Engineering, UK. \\ ${ }^{2} \mathrm{AWE}$, Aldermaston, UK.
}

\begin{abstract}
The paper presents an experimental investigation into the internal blast loading of open-ended, seamless, mild steel cylinders. A series of 10 trials were conducted on explosively loaded vessels using increasing masses of PE4 explosive. The objective of these trials was to determine the maximum circumferential strain induced in the cylinder wall as a result of the blast loading and also to determine the minimum amount of explosive required to cause wall failure in the cylinder. A cylindrical-shaped charge was detonated at the centre of the cylinder as this was more likely to produce a symmetrical blast wave than a spherical-shaped charge. The response of the cylinder with increasing charge size goes from large plastic deformation to failure by propagation of longitudinal cracks in the region of localized wall thinning. It is thought that the localized wall thinning is a result of unstable modal vibration and this is confirmed by instability analysis. This investigation has allowed insight into the failure process of these structures not previously examined under the given loading conditions. The data generated in these trials has been used successfully to validate numerical and theoretical models of the cylinder response to impulsive loading. Keywords: Cylinder, explosive, fracture, impulsive, modal instability, plastic deformation.
\end{abstract}

\section{INTRODUCTION}

Much research has been conducted on the explosive loading of spherical and cylindrical vessels with a view to analysing both the elastic and the plastic responses of these structures to the applied loading. In some instances, where the structure is packed with explosive, failure of the vessels has been observed and analysed [1-5]. However, to date no research has been discovered on characterizing the failure mechanism of a vessel in which the explosive is not in direct contact with the vessel wall at detonation. In the case where structure and explosive are in direct contact, the material behaviour is governed by transmission of the detonation shockwave through the material. In this investigation, detonation produces a shockwave that propagates in air and interacts with the structure at close range. An open-ended cylinder configuration was chosen for these trials to minimize multiple reflections of the shockwave inside the vessel and to ensure the response was impulsive with no influence from residual quasi-static overpressure. Some details of the background to the project, preliminary results and the analytical approach are given in [6]. The purpose of this paper is to explore in more detail the character of the failure process of explosively loaded open-ended steel cylinders with a full set of results which has allowed insight into the failure process of these structures not previously examined under the given loading conditions.

The purpose of the experiments presented in this paper is to determine the failure mechanism of open-ended, thin-walled cylinders under explosive loading by a centralized charge. The results of these experiments can be used to validate theoretical and numerical simulations when determining the plastic circumferential strain in the cylinder wall and help with the design of vessels that are used to confine or contain the high transient pressures generated by detonating explosives. The experiments discussed in this paper were conducted at the Health and Safety Laboratory, Buxton, UK. 


\section{EXPERIMENTAL SETUP}

This section presents the setup and experimental procedure for generating the blast loading of the open-ended, thin-walled cylinders. A series of 10 tests were conducted on the cylinders using increasing charge masses of PE4 high explosive. The purpose of these tests was to determine the minimum charge mass required to cause wall failure in the cylinders and also to characterize the failure mechanism. Failure in this case was defined as the throughthickness fracture of the cylinder wall.

Attempts were made to measure strain history directly from gauges attached to the outer surface of the cylinder at the charge location but due to the very high accelerations the gauges failed to remain attached and produced no useable data. Pressure gauges were mounted at both ends of the cylinder to measure the incident free-field pressure near the exit and this gave a qualitative measure of the symmetry of loading inside the cylinder by comparing the two gauge outputs. However, final cylinder circumference and wall thickness were the most useful measurements recorded in the tests. The cylinders were secured in the horizontal position by a sling and V-shaped support as shown in Fig. 1. The location of the pressure gauges (Kulite ETS-1A-375-500 SG) is also shown in Fig. 1.

\subsection{Test specimens}

The test specimens were cut from two lengths of $6 \mathrm{~m}$ long, seamless pipe section made from API 5L X-42 mild steel. All of the experimental pipe specimens had a nominal inner radius of $152.4 \mathrm{~mm}$, wall thickness of $9.5 \mathrm{~mm}$ and length of $800 \mathrm{~mm}$.

\subsection{Charge size and geometry}

A cylindrical charge shape was used to obtain near axi-symmetric loading on the cylinder wall. Spherical charges were unlikely to produce a uniform blast wave due to the difficulty in centralizing the detonator and the detonator dimensions influencing the profile of the

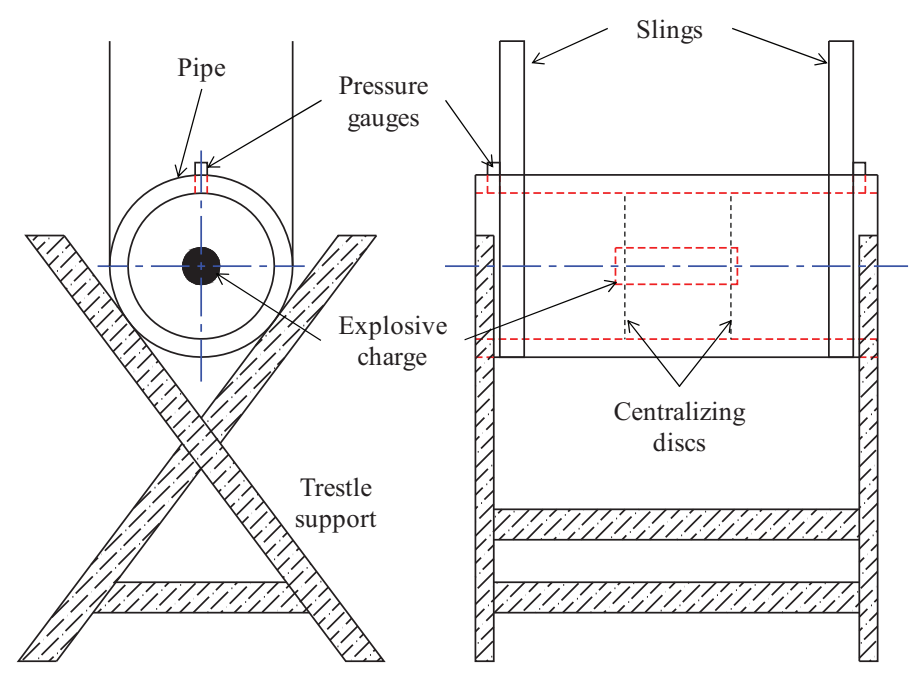

Figure 1: Experimental setup of cylinder tests. 
generated blast wave. Detonators were placed at the centre of each circular face of the cylindrical charge to be detonated simultaneously and this ensured that the blast wave was near symmetric. This configuration caused a collision of the blast waves, propagating towards each other from the detonated surfaces, at the centre of the charge that would propagate radially and axi-symmetrically towards the vessel wall.

All the charges were prepared according to a length to diameter ratio of 1 . The minimum charge size is limited by the critical detonation diameter of the explosive, below which the explosive would be unable to sustain self-propagation [7]. PE4 was used as the explosive material as it has a relatively low sensitiveness, so there was little risk of it detonating without the initiation of a detonator. Being a plastic explosive, it is also a malleable substance making it easy to mould into charges of the correct size and shape required in this series of trials. The critical detonation diameter of PE4 is between 3.81 and $5.08 \mathrm{~mm}$ [8]. The explosive has a density of $1600 \mathrm{~kg} / \mathrm{m}^{3}$ so, theoretically, the minimum mass of explosive that could be used in this series of trials was approximately $0.16 \mathrm{~g}$. The maximum mass of PE4 that could be used was constrained by the internal diameter of the pipe. The purpose of these tests was to cause deformation of the pipe up to the point of failure without contact between the explosive and the vessel wall.

The cylindrical charge was located in the geometric centre of the pipe with coincident centre axes. To locate the charge in the geometric centre of the vessel, the required mass of PE4 was shaped and wrapped in thick brown paper to maintain the dimensions and shape. Two circular cardboard collars were cut so that the outer diameter was equal to the inner pipe wall diameter and the inner diameter was equal to the cylindrical charge diameter. This whole arrangement could be fitted inside the cylinder and ensured accurate location of the charge as shown in Fig. 1. The high temperatures involved in the detonation process instantaneously vaporized the cardboard supporting the explosive.

\subsection{Instrumentation}

The pressure gauges used in the trials were Kulite ETS-1A-375-500 SG pressure transducers. These gauges were designed by Kulite for blast pressure applications. Features of this gauge included a very high natural frequency, operating temperatures in the range from $-55^{\circ} \mathrm{C}$ to $+120 \mathrm{C}$ and a maximum burst pressure of $280 \mathrm{MPa}$. The sensor of the gauge was covered with a mixture of silicon grease, photocopy toner and various other chemicals, which served to protect the gauges from the radiation produced by the detonating explosive.

The recording of the pressure was carried out using either a Nicolet Odyssey OD-200 or a Nicolet Odyssey XE data logger. Both these data acquisition systems are Windows-based data recorders with a transient data capture frequency of up to $10 \mathrm{MHz}$. The Nicolet Odyssey XE was set to capture data at a frequency of $1 \mathrm{MHz}$ and the Nicolet Odyssey OD-200 at a frequency of $500 \mathrm{kHz}$. Both recorders had a large data storage capacity to allow the transient event to be captured within a relatively long timeframe. This capability enabled the recorders to be armed and running and the charge to be fired at a safe distance.

The main concern with the detonation of the explosive was the ability to achieve simultaneous ignition of the two detonators on every charge, to produce a uniform, axi-symmetric blast wave. Calculations were made on the possibility of non-simultaneous detonation of the detonators used in the series of tests. The L2A1 militarized detonators that were considered to have a break-through time of $80 \pm 40 \mu \mathrm{s}$. If the worst case is assumed and there was a difference of $80 \mu \mathrm{s}$ in the break-through time between the two detonators, given that the velocity 


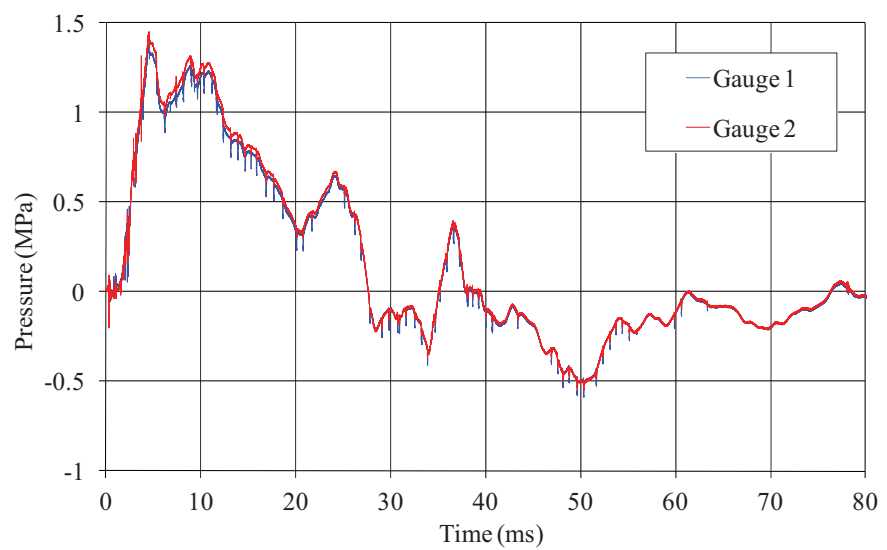

Figure 2: Recorded pressure data for the blast waves exiting the pipe generated by the detonation of $1200 \mathrm{~g}$ PE4.

of detonation of PE4 is $8193 \mathrm{~m} / \mathrm{s}$ [9] and the maximum charge length was $106 \mathrm{~mm}(1.5 \mathrm{~kg}$ PE4 charge), then the shockwave would propagate along the length of the cylindrical charge before the second detonator fired. This calculation was also validated using finite element analysis. Precautions were therefore taken to achieve simultaneous detonation by linking the detonators to the fuse box using equal lengths of wire from the same batch of wiring. Detonators were also used from within the same batches and from the same supplier to minimize any differences between them in the manufacture.

\subsection{Pressure gauge output}

Due to a series of pressure gauge failures, the only useful pressure data was recorded in tests with $1000 \mathrm{~g}$ and $1200 \mathrm{~g}$ charges. The pressure history of both gauges (located near the exit of the cylinder) for the test with a $1200 \mathrm{~g}$ charge is given in Fig. 2. The graphs of both gauges almost overlap, indicating near simultaneous detonations and gave confidence in the procedure to ensure uniform radial loading of the pipe wall. High speed video also demonstrated this through the disturbance of the lacquer that coated the outer surface of the pipe. It should be noted that the pressure magnitudes in Fig. 2 are much lower than the pressures generated by the explosive charge at the inside surface of the cylinder at mid-length. A simulated pressure loading profile is given in Fig. 13 in Section 4 in which the peak pressure is 2 orders of magnitude higher than that in Fig. 2 and the duration is significantly shorter, $0.2-0.3 \mathrm{~ms}$ with a rise time of the order of $10 \mathrm{~s}$ of micro-seconds. The recorded pressure-time histories here were only used to check for symmetrical pressure loading at each end of the vessel.

\section{STRUCTURAL RESPONSE}

The series of trials on the mild steel pipes resulted in permanent deformation of the cylinder wall in the form of a large circumferential bulge in the middle of the specimen, the location of the HE charge detonation wave collisions as shown in Fig. 3. Some of the specimens failed by rupture in the wall thickness when the charge mass was of sufficient magnitude as shown in Fig. 4. One of the aims of the test series was to determine the 


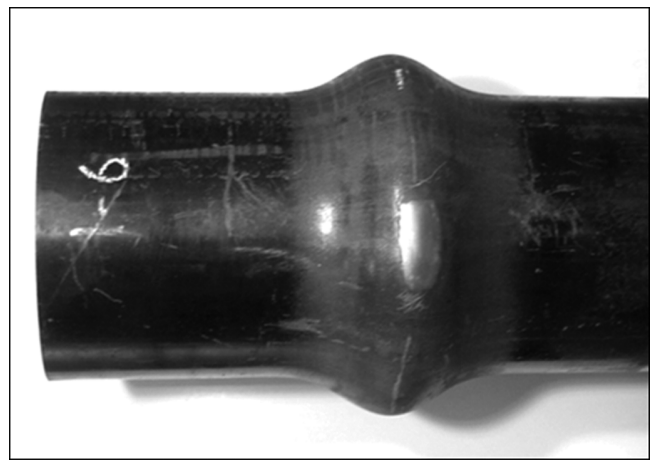

Figure 3: Cylinder wall deformation (1200 g HE charge).

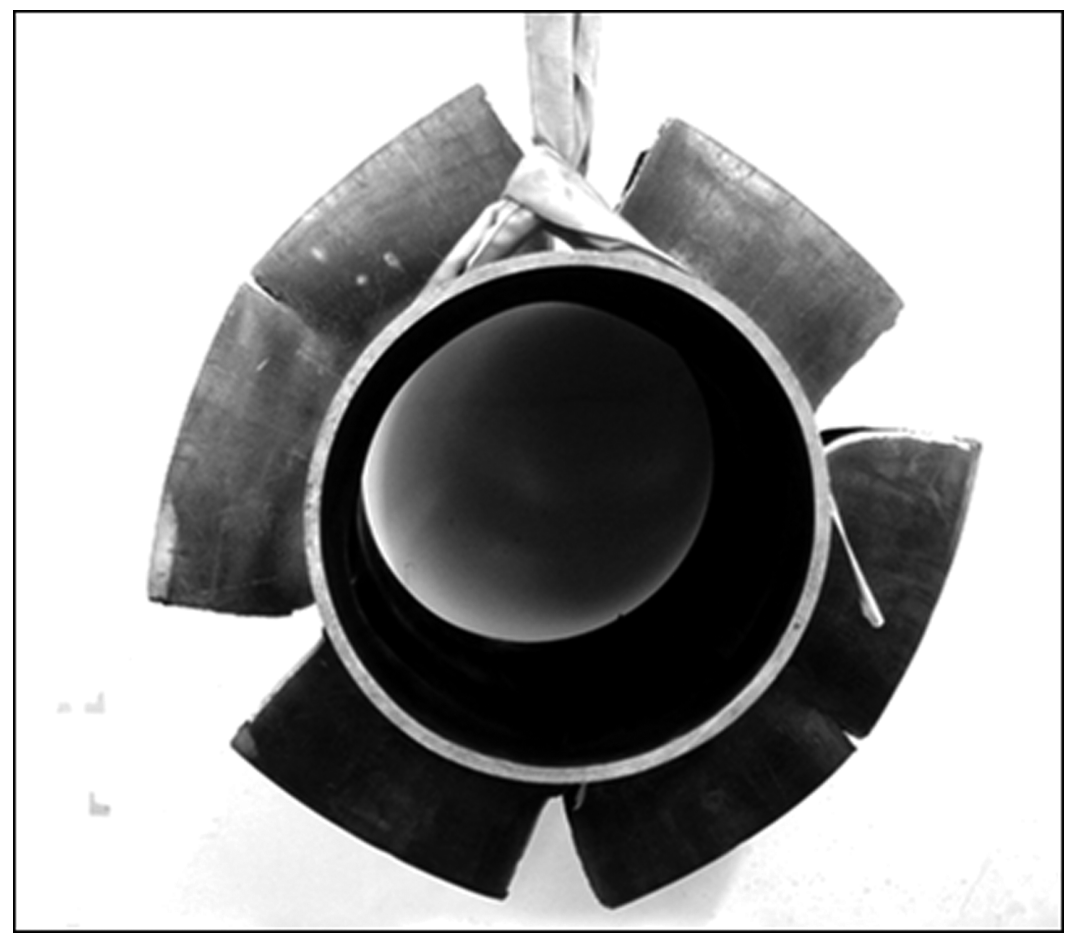

Figure 4: Fractures in cylinder (1500 g HE charge).

minimum charge mass required to cause wall failure of the cylinder. This was achieved by increasing the charge mass in steps of $200 \mathrm{~g}$ initially until a rupture occurred. With only a limited number of specimens to work with, the first test began at a charge size of $600 \mathrm{~g}$. Table 1 shows the order of the tests. After test \#4, the charge mass was increased by $300 \mathrm{~g}$ and produced catastrophic failure as is shown in Fig. 4. After test \#5, the charge size was reduced in smaller increments down to $1225 \mathrm{~g}$ charge size. This process enabled the critical charge mass to cause failure to be determined to the nearest $25 \mathrm{~g}$ of PE4. A repeat of the $1200 \mathrm{~g}$ charge test was carried out to check consistency. It can be seen from Table 1 that no 
Table 1: Sequence of experiments and pipe response.

\begin{tabular}{ccccc}
\hline Test \# & Pipe ref. & PE4 charge mass $(\mathrm{g})$ & $\begin{array}{c}\text { Circumference } \\
\text { measured }(\mathrm{m})\end{array}$ & $\begin{array}{c}\text { Bulk circumferential } \\
\text { strain }\end{array}$ \\
\hline 1 & $1-4$ & 600 & 1.138 & 0.119 \\
2 & $1-2$ & 800 & 1.200 & 0.180 \\
3 & $1-6$ & 1000 & 1.278 & 0.256 \\
4 & $2-12$ & 1200 & 1.352 & 0.329 \\
5 & $2-14$ & 1500 & - & Rupture \\
6 & $2-16$ & 1300 & - & Rupture \\
7 & $1-10$ & 1250 & - & Rupture \\
8 & $2-18$ & 1200 & 1.333 & 0.310 \\
9 & $1-8$ & 1225 & - & Rupture \\
10 & $2-20$ & 500 & 1.104 & 0.085 \\
\hline
\end{tabular}

rupture occurred, but the hoop strain was marginally ( 6\%) lower than test \#4. The final test with the last specimen was carried out at $500 \mathrm{~g}$ charge size with high speed digital photography.

\subsection{Cylinder wall deformation}

Charge sizes in the range of 500-1200 g produced large wall deformation in the form of a uniform radial bulge at the mid-span of the cylinder pipe specimen (tests \#1-4 and tests \#8 and \#10). The extent of the bulge along the length of the pipe coincides approximately with the length of the cylindrical HE charge and is indicated clearly on the outside surface of the vessel by the damage to the surface coating of the vessel as shown in Fig. 3. This coating damage first appears in the form of two rings on either side of the bulge for charge sizes under $800 \mathrm{~g}$. Above $800 \mathrm{~g}$ charge size, the coating damage extends across the whole bulge. The coating damage was also clearly seen in the high speed camera images for test \#10 (500 g HE charge) during the first $1500 \mu$ s of the blast process.

\subsection{Wall thinning}

As the pipe expands radially and the circumferential strain increases in the region of the bulge, the wall thickness in the expanding material decreases (for the volume to remain constant by conservation of mass). This is referred to as the bulk material wall thickness in Fig. 5. Closer inspection of the wall damage revealed that in some of the vessels, localized thinning or necking of the vessel wall occurred in some regions around the bulge at the midlength where maximum circumferential deformation occurred. This is referred to as the wall thickness in thinned regions in Fig. 5. It was thought that this was due to instabilities in the mode 6 circumferential vibration of the pipe which gave rise to 12 regions where thinning or a combination of thinning and fracture could occur.

The magnitude of the circumferential deformation in the cylinder wall increased with charge size. When the charge size exceeded $1000 \mathrm{~g}$ HE mass, the localized regions of wall thinning due to the unstable circumferential vibration were observed. These occur at the peak amplitude of the vibration. Tensile fracture occurred at around $1225 \mathrm{~g}$ charge mass in the 


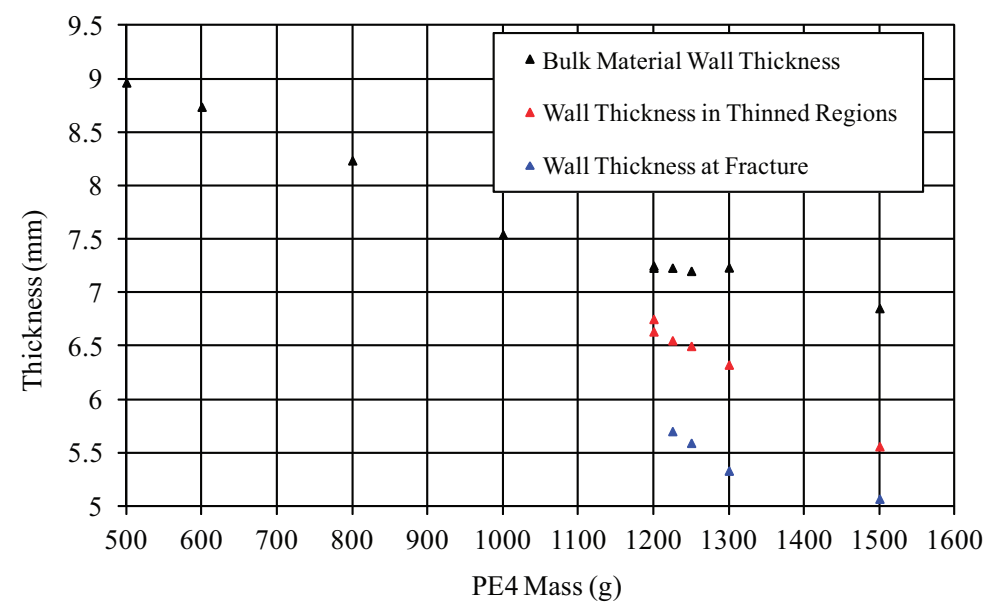

Figure 5: Wall thickness in the deformed and failed cylinder specimens.

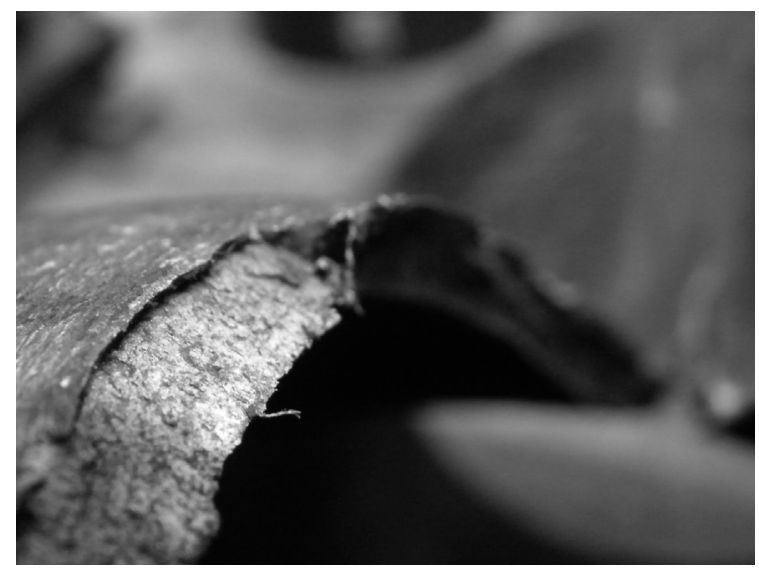

Figure 6: Fracture surface in failed cylinder in test with $1250 \mathrm{~g}$ PE4 charge.

regions of localized wall thinning. Closer inspection of the fracture surface showed typical shear failure of the fracture surface as shown in Fig. 6. The wall thickness at the point of failure was also measured and this is referred to in Fig. 5 as the wall thickness at fracture.

It is observed from Fig. 5 for charge masses 1225-1300 g that fracture does not appear to occur at a constant wall thickness. The bulk material wall thickness remains constant across this range of charge mass but both localized wall thickness and the wall thickness at the point of fracture decrease with increasing charge mass and hence circumferential strain. Deformation becomes more localized due to the higher stress in the thinned regions. It can also be seen that for a charge mass of $1500 \mathrm{~g}$ the wall thickness in a region of localized wall thinning is less than the wall thickness at the point of fracture in the tests with $1225 \mathrm{~g}$ and $1250 \mathrm{~g}$ charges. This suggests that the magnitude of wall thinning alone does not govern when fracture occurs, for example, defects in the microstructure of the material. It is also noted that very large bending deformation will cause localized wall thinning in the region of very high 
bending strain. The circumferential (engineering) strains at failure in the blast tests ranged from about 32 to $33 \%$. Elongation at the static UTS in the material characterization tests ranged from 15 to $20 \%$ true strain.

\subsection{Failure characteristics}

Test \#5 with $1500 \mathrm{~g}$ of HE charge caused failure through the wall thickness of the cylinder. This failure was characterized by six longitudinal fractures originating at the mid-length of the vessel approximately equispaced around the circumference. Propagation of the cracks led to collapse of the vessel about a plastic hinge around the circumference between the fracture surfaces as shown in Fig. 4. The uniform spacing of the fracture zones around the vessel circumference suggests that a dynamic vibration phenomenon is at work to precipitate failure. It was considered that an unstable dynamic mode of vibration (in this case mode 6) led to simultaneous multiple fracture initiation at the peak amplitude of vibration. The half-period mode 6 vibration modes for a cylinder are illustrated in Fig. 7.

Regions of localized wall thinning were detected at points mid-way between the fracture locations as shown in Fig. 8. This observation further supported the theory that the mode 6 circumferential unstable vibrations initiated failure after a number of cycles. In successive cycles the strain grows with increasing amplitude of vibration to a critical value upon which fracture occurs. A total of 12 regions of wall thinning including fracture points were detected in all the tests to rupture, that is, test \#5-7 and test \#9. Furthermore, the spacing of these regions was almost equidistant around the vessel circumference in each case which again provides further experimental evidence for the theory of unstable mode 6 vibrations excited by the loading. Figure 9 gives the location of fracture initiation points (indicated by red dots) in the tests \#5, 6 and 9 leading to rupture.

Evidence of unstable adiabatic shear being the failure mechanism of the blast loaded pipes was observed in the microstructure of the fracture surfaces made evident by the distortion of the grains in the fracture vicinity. A number of authors have previously characterized the failure in explosively driven cylinders as being due to adiabatic shear resulting from localized temperature increases. These temperature increase cause a highly localized thermal softening of the material which leads to large stresses along certain shear plains and leads to fracture. All of the fractures were inclined at angles of approximately $45^{\circ}$ to the cylinder centreline where it is presumed that the shear planes lie.
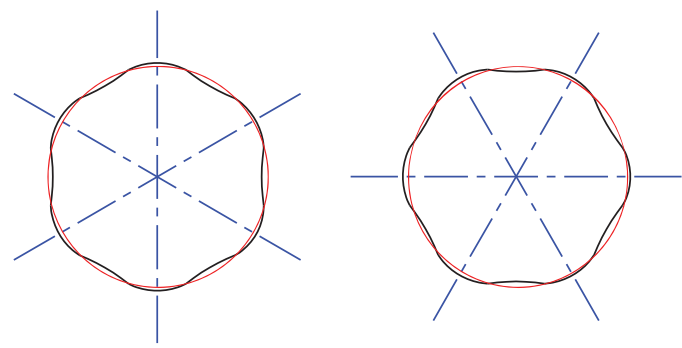

Figure 7: Half-period mode 6 vibration modes for a cylinder under internal pressure loading. 


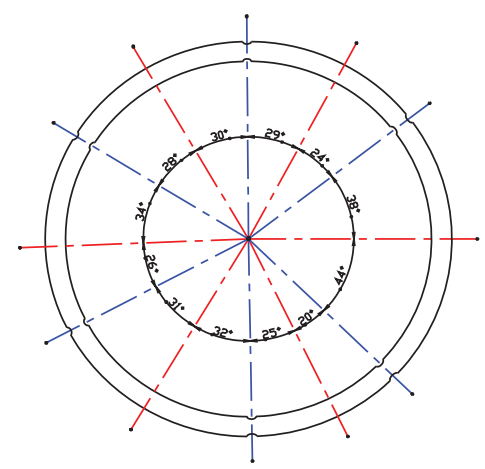

Figure 8: Fracture locations (red lines) and regions of localized wall-thinning between fracture locations (blue lines) for test \#5 (1500 g PE4).

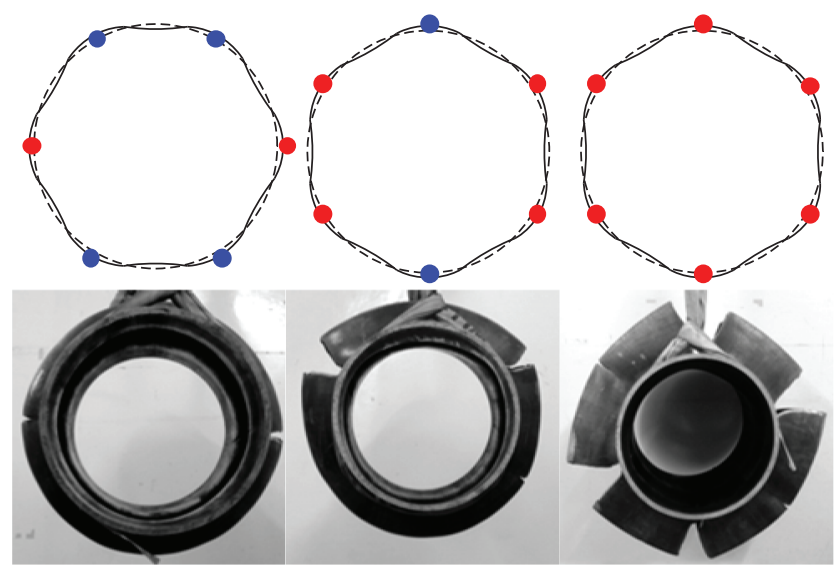

Figure 9: Points of maximum amplitude/tensile strain (red and blue dots) along with fracture locations (red dots) for tests \#9, 6 and 5 (from left to right).

\subsection{Vibration instability analysis}

A method for predicting the stable and unstable vibration modes for a uniform cylindrical shell under impulsive pressure using a Mathieu stability analysis involving two sets of dimensionless parameters is presented in Goodier and McIvor [10]. In this, the shell remains elastic and the ensuing motion is a free vibration in the plane of the cross-section, a simple in-and-out 'breathing' mode. Imperfections in the uniformity of the circular cross-section cause flexural motion to develop through interaction between the circumferential compressive (or tensile) stress and the flexural curvature. It is suggested that certain types of flexural motion can strongly excite critical growth in amplitude of the cyclic vibrations. While external uniform impulse loading is implied in the paper, the authors consider the analysis to be equally applicable to internal uniform impulse loading due to the similarity of the 'breathing mode' produced by an internal explosion loading. This approach was used to analyse the test cylinders that failed and to verify the conjecture that the uniform crack spacing around the 
mid-length circumference of the impulsively loaded cylinder was the result of localized increase in the circumferential strain due to modal vibration instability.

The vibration analysis uses Mathieu stability theory for the prediction of stable and unstable circumferential vibration modes. This is expressed in periodic form as $\ddot{a}_{n}+\left(\Omega_{n}-\mu_{n} \sin \tau\right) a_{n}=0$ for $n \geq 2$, where $\ddot{a}_{n}$ is the radial acceleration of the cylinder, $a_{n}$ is the radial displacement and is the dimensionless time parameter. The dimensionless parameters $\Omega_{n}=n^{2} a^{2}\left(n^{2}-1\right)^{2} /\left(n^{2}+1\right)$ and $\mu_{n}=n^{2} v_{0}\left(n^{2}-2\right) /\left(n^{2}+1\right) c$ describe the thickness of the cylinder wall and the initial impulse velocity, respectively, for each vibration mode, where $\mathrm{n}$ is the mode number, $\mathrm{v}_{0}$ is the initial velocity, $\mathrm{c}=\sqrt{\mathrm{E} / \rho\left(1-v^{2}\right)}$ is the elastic wave velocity through the material where E is Young's modulus for the material, is Poisson's ratio, is the density and $a^{2}=\mathrm{h}^{2} / 12 \mathrm{R}_{\mathrm{m}}{ }^{2}$ is the thickness parameter, where $\mathrm{h}$ is the wall thickness and $\mathrm{R}_{\mathrm{m}}$ is the mean radius of the cylinder. The stability analysis was based on the measured values in test \#4 where it is assumed that the pipe was at the limit of failure initiation, maximum strain of approximately 0.33 , corresponding to a measured outer diameter of $435 \mathrm{~mm}$ and wall thickness of $7.25 \mathrm{~mm}$.

An initial velocity of $250 \mathrm{~m} / \mathrm{s}$ (Fig. 14) was determined from a numerical analysis of test \#4 as mentioned in the next section. This parameter, along with the material parameters for Young's modulus, Poisson's ratio and the material density, determined from material characterization tests, were input into the above equations for the first 10 circumferential vibration modes. The results of this are given in Table 2.

The results in Table 2 were mapped onto a Mathieu stability diagram for thin cylindrical shells to obtain the graph shown in Fig. 11 where the shaded areas represent regions of stability and the unshaded areas represent regions of instability. From Fig. 10 it can be seen that, at an impulse velocity of $250 \mathrm{~m} / \mathrm{s}$, the circumferential vibration modes 4 to 10 are all unstable. A further analysis performed by Goodier and McIvor [10] was to determine the critical unstable mode in a vibrating system $\left(n=n_{c}\right)$ by determining which mode would lie on the line $\Omega_{\mathrm{n}}=1 / 4$. The first unstable region in the stability analysis lies on the line $\Omega_{\mathrm{n}}=1 / 4$, where it is guaranteed to be unstable. The critical unstable mode was calculated by applying $\Omega_{\mathrm{n}}=1 / 4$ to

Table 2: Mathieu stability analysis results for test \#4 loaded with an initial impulse velocity of $250 \mathrm{~m} / \mathrm{s}$.

\begin{tabular}{lll}
\hline Mode number & $\Omega_{\mathrm{n}}$ & $\mu_{\mathrm{n}}$ \\
\hline 0 & 0 & 0 \\
1 & 0 & 0.023 \\
2 & 0.001 & 0.074 \\
3 & 0.006 & 0.292 \\
4 & 0.020 & 0.610 \\
5 & 0.053 & 1.024 \\
6 & 0.115 & 1.532 \\
7 & 0.217 & 2.133 \\
8 & 0.376 & 2.827 \\
9 & 0.608 & 3.614 \\
10 & 0.932 & 4.493 \\
\hline
\end{tabular}




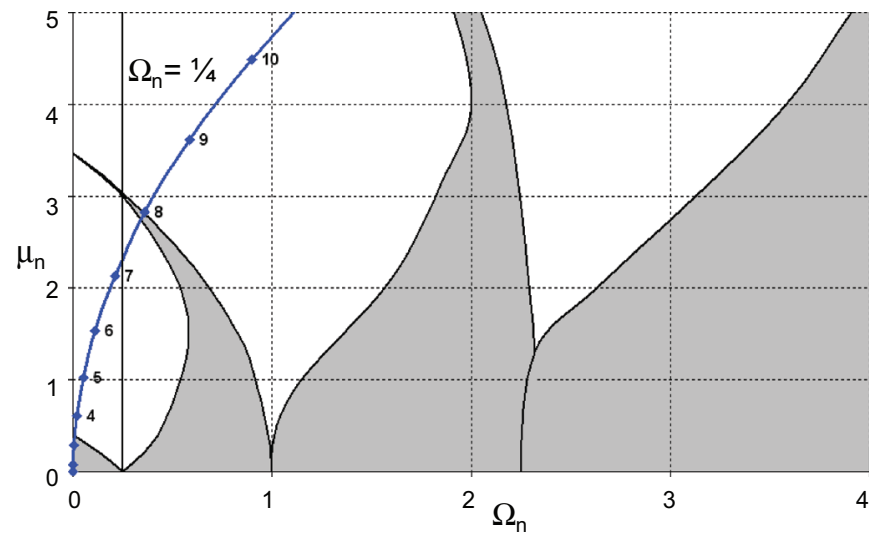

Figure 10: Mathieu stability diagram with mapped results of stability analysis for test \#4 loaded with an initial impulse velocity of $250 \mathrm{~m} / \mathrm{s}$.

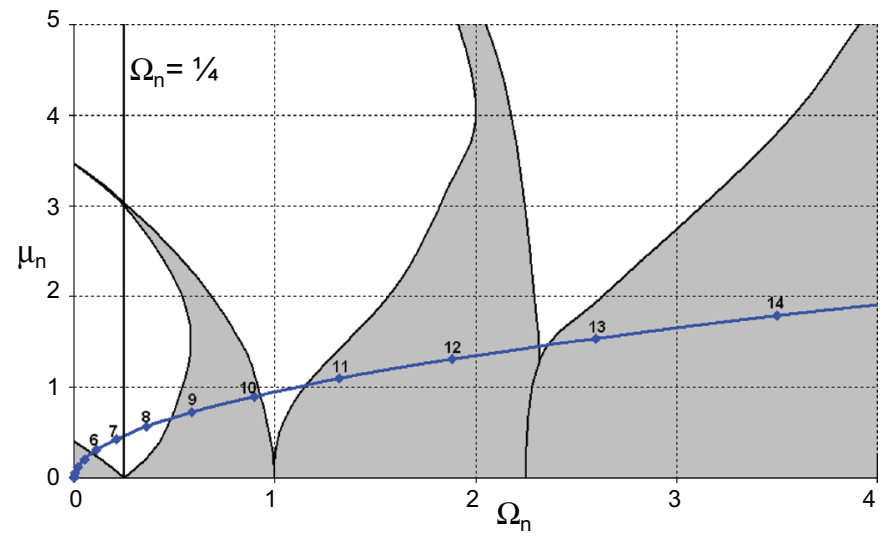

Figure 11: Mathieu stability diagram with mapped results of stability analysis for test \#4 loaded with an initial impulse velocity of $50 \mathrm{~m} / \mathrm{s}$.

$\Omega_{\mathrm{n}}=\mathrm{n}^{2} \alpha^{2}\left(\mathrm{n}^{2}-1\right)^{2} /\left(\mathrm{n}^{2}+1\right)$ and gave a critical mode of 7 (to the nearest whole number). It is concluded that the mode 6 instability conjecture is therefore plausible given that the Goodier and McIvor approach applies to elastic response of cylindrical shells and not plastic response. As a comparison, a further vibration analysis was performed for the same shell but with a reduced initial impulse velocity of $50 \mathrm{~m} / \mathrm{s}$ to determine the effect of the magnitude of initial impulse velocity on the stability of the cylinder. The results are given in Fig. 11.

From Fig. 11 it can be seen that there are fewer unstable modes of circumferential vibration in the cylinder when the magnitude of the initial impulse velocity is smaller. In the elastic analysis for a velocity of $50 \mathrm{~m} / \mathrm{s}$, the results show only modes 6 to 8 to be unstable. Mode 7, the critical mode, will always remain in an unstable region for a cylinder with the dimensions of those analysed because it will always lie on the same $\Omega_{\mathrm{n}}$ value which is governed by $\Omega_{\mathrm{n}}=\mathrm{n}^{2} \alpha^{2}\left(\mathrm{n}^{2}-1\right)^{2} /\left(\mathrm{n}^{2}+1\right)$. 


\section{VALIDATION OF NUMERICAL ANALYSIS}

Finite element (FE) numerical models were validated against the data presented in this paper. The computational simulations and the experimental measurements agree well as shown in Fig. 12. The numerical models were constructed and solved using a 2D axi-symmetric solver in ANSYS AUTODYN. The explosive charge and surrounding air were modelled using an Euler grid and the steel shell was modelled using a Lagrange mesh. Static and dynamic material characterization was carried out for the cylinder material, and curve fits for the data were applied to Cowper-Symonds and Johnson-Cook constitutive equations. These strain rate dependant constitutive models generated peak strain rates in the FE simulations up to about $1700 \mathrm{~s}^{-1}$ for an explosive charge of $1.2 \mathrm{~kg}$. The increase in peak strain rate with charge size was near linear from about $200 \mathrm{~s}^{-1}$ at $0.2 \mathrm{~kg}$ charge size. The static mechanical properties of the steel and the derived strength model parameters as used in the numerical analyses are summarized in Table 3. This data was obtained from longitudinal tensile test specimens machined from the wall section of the same pipe material used for the explosive tests.

In addition to applying a strength model to define the material response to blast loading, a model is also necessary to determine the pressures and impulses produced by the detonation of the PE4 plastic explosive. The explosive is modelled by replacing elements of the air in the Euler grid with those of PE4, which are defined by an equation of state. Such models to characterize explosives include the Lee-Tarver model and the Jones-Wilkinson-Lee (JWL) model. The PE4 model applied in this analysis uses the JWL equation of state, which mathematically models the pressure-volume-energy behaviour of the detonation products in metal acceleration applications. This model is expressed by eqn (1), where $\mathrm{P}$ is the pressure, $\mathrm{A}_{\mathrm{JWL}}, \mathrm{B}_{\mathrm{JWL}}, \mathrm{R}_{1}, \mathrm{R}_{2}$ and $\mathrm{W}$ are experimentally determined constants, $\mathrm{V}$ is the volume of the detonation products in relation to the volume of the undetonated explosive and $\mathrm{E}^{*}$ is the energy per unit volume.

$$
P=A_{J W L}\left(1-\frac{W}{R_{1} V}\right) e^{-R_{1} V}+B_{J W L}\left(1-\frac{W}{R_{2} V}\right) e^{-R_{2} V}+\frac{W^{*}}{V}
$$

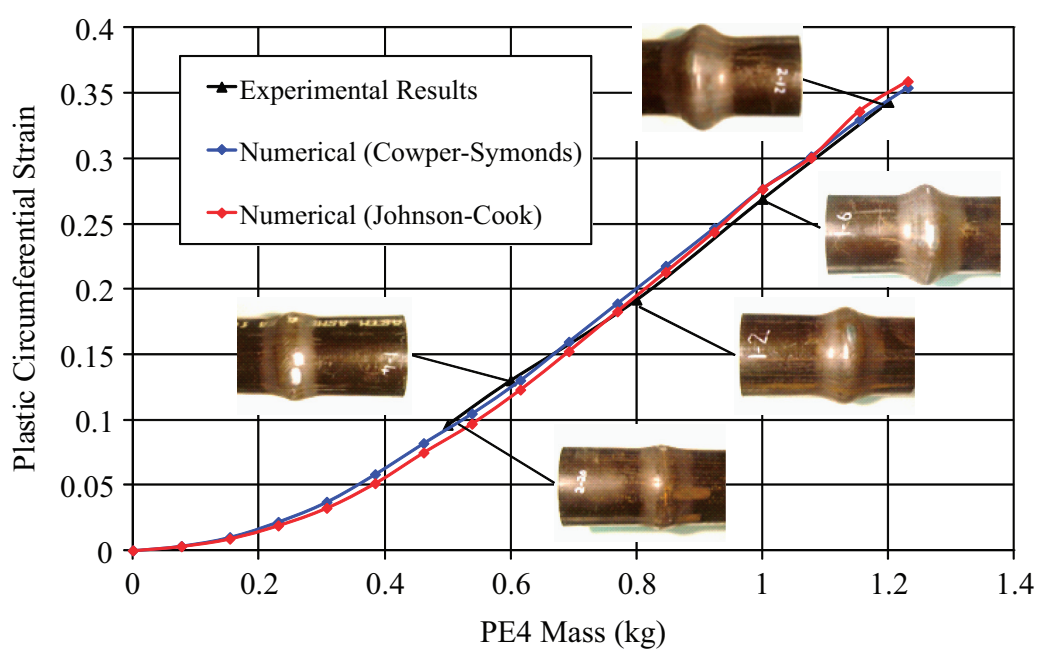

Figure 12: Comparison between finite element numerical and experimental results for the mid-length circumferential strain in open-ended mild steel pipes. 
Table 3: Dimensions and material properties for the steel cylinders.

\begin{tabular}{lll}
\hline Dimensions & & \\
Outer diameter & 323.8 & $\mathrm{~mm}$ \\
Wall thickness & 9.5 & $\mathrm{~mm}$ \\
Length & 800 & $\mathrm{~mm}$ \\
Static material properties & & \\
Density & 7723 & $\mathrm{~kg} / \mathrm{m}^{3}$ \\
Yield stress & 314.4 & $\mathrm{MPa}$ \\
Young's modulus & 207 & $\mathrm{GPa}$ \\
Poisson's ratio & 0.28 & \\
UTS & 554.6 & $\mathrm{MPa}$ \\
Elongation at UTS & 16 & $\%$ \\
Cowper-Symonds parameters & & \\
Yield stress & 314.4 & $\mathrm{MPa}$ \\
Hardening constant & 590.0 & $\mathrm{MPa}$ \\
Hardening exponent & 0.47 & \\
Strain rate constant (D) & 289.2 & $\mathrm{~s}^{-1}$ \\
Strain rate exponent (q) & 4.053 & \\
Johnson-Cook parameters & & \\
Yield stress (A) & 395.0 & $\mathrm{MPa}$ \\
Hardening constant (B) & 590.0 & $\mathrm{MPa}$ \\
Hardening exponent (n) & 0.47 & \\
Strain rate constant (C) & 0.024 & \\
Thermal softening exponent (m) & 1.03 & \\
Reference strain rate $(\dot{\varepsilon})$ & 1 & $\mathrm{~s}^{-1}$ \\
\hline
\end{tabular}

Table 4: JWL parameters for modelling the PE4 explosive in Autodyn.

\begin{tabular}{ll}
\hline Reference density & $1600 \mathrm{~kg} / \mathrm{m}^{3}$ \\
Parameter A & $718.16 \mathrm{GPa}$ \\
Parameter B & $11.85 \mathrm{GPa}$ \\
Parameter R1 & 4.7 \\
Parameter R2 & 1.3 \\
Parameter W & 0.25 \\
C-J detonation velocity & $8.18 \times 10^{3} \mathrm{~m} / \mathrm{s}$ \\
C-J energy/unit volume & $8.12 \times 10^{6} \mathrm{~kJ} / \mathrm{m}^{3}$ \\
C-J pressure & $26.765 \mathrm{GPa}$ \\
Others & 0 \\
Auto-convert to ideal gas & Yes \\
\hline
\end{tabular}

In a similar way to determining the Johnson-Cook material model constants, the parameters of the JWL equation of state are obtained by modifying the constants in eqn (1) to best fit an actual PE4 explosive pressure loading. These values are given in Table 4. 
Table 5: Cut-off parameters for modelling the PE4 explosive in Autodyn.

\begin{tabular}{ll}
\hline Maximum expansion & 0.1 \\
Minimum density factor (Euler) & $1.0 \times 10^{-6}$ \\
Minimum density factor (SPH) & 0.2 \\
Maximum density factor (SPH) & 3.0 \\
Minimum sound speed & $1.0 \times 10^{-6} \mathrm{~m} / \mathrm{s}$ \\
Maximum sound speed & $1.01 \times 10^{20} \mathrm{~m} / \mathrm{s}$ \\
Maximum temperature & $1.01 \times 10^{20} \mathrm{~m} / \mathrm{s}$ \\
\hline
\end{tabular}

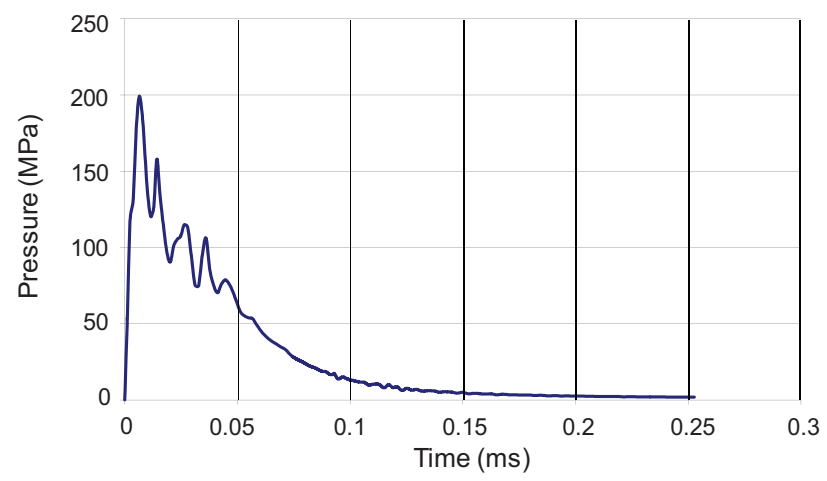

Figure 13: FE generated pressure loading for a $0.54 \mathrm{~kg}$ PE4 spherical explosive charge.

The 'Auto-convert to ideal gas' option indicates that the gaseous products are modelled using ideal gas equations once the detonation process has been completed. Further parameters that are included in the modelling were the 'cut-off' parameters, which are shown in Table 5.

An example of a pressure pulse generated by the detonation of a spherical PE4 charge in the Autodyn analysis is shown in Fig. 13 for $0.53 \mathrm{~kg}$ of explosive. This pulse shape is characterized by an almost instantaneous rise to peak pressure followed by an exponential decay. The pressure profile in Fig. 13 shows a number of pressure peaks occurring during the exponential decay of the pulse. When a spherical explosive detonates, a shockwave is produced, which travels radially outwards at a greater velocity than the expanding gas products of the chemical reaction within the explosive. Pressure profiles, such as the one in Fig. 13, result from the shockwave interaction with a structure. The pressures produced by the expanding gases are far smaller in magnitude. Since the shockwave travels faster, it reaches and reflects off the structure before the expanding gas behind it. This gas is very dense and causes another reflection of the shockwave, this time back in the direction of the structure where it manifests itself as a peak during the decay stage of the pressure profile. This phenomenon has previously been analysed by Karpp et al. [11] when numerically analysing the responses of containment vessels to blast loading.

Figure 14 shows the transient velocity profile generated by FE analysis for the explosively loaded cylinder at mid-length when loaded by a $1.2 \mathrm{~kg}$ cylindrical PE4 explosive charge in Autodyn.

Further details of the numerical analysis are given in Rushton et al. [6]. 


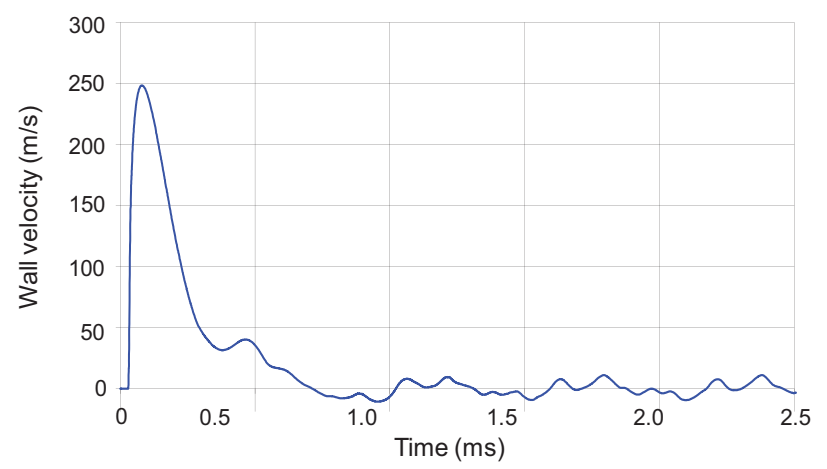

Figure 14: Simulated wall velocity for a $1.2 \mathrm{~kg}$ cylindrical PE 4 charge.

\section{CONCLUDING REMARKS}

A series of field trials on explosively loaded, open-ended, thin-walled, mild steel cylinders has allowed insight into the failure process of these structures not previously examined under the given loading conditions. The data generated in these trials has been used successfully to validate numerical and theoretical models of the vessel response to impulsive loading. The failure process can be summarized as follows:

(a) Internal explosive loading causes the cylinder to expand radially mid-length and at the same time oscillate about a mean expanding circumference.

(b) The cylinder wall deforms plastically through a combination of radial expansion and oscillation that result in bulk wall thinning and equally spaced regions of localized wallthinning around the cylinder circumference. The uniform spacing and number of regions indicate an unstable modal vibration confirmed by instability analysis.

(c) Further plastic circumferential straining and radial oscillations results in the initiation of through-wall fractures in the region of localized thinning by the so-called adiabatic shear process previously characterized by other researchers. The fracture surface is inclined at approximately $45^{\circ}$ to the centreline of the cylinder.

(d) The energy required to initiate fracture is greater than the energy required to cause fracture propagation; so once fractures initiates, the cracks propagate rapidly in a longitudinal direction.

(e) When the energy level is sufficiently high, the vessel collapses in an unstable manner in the middle about a plastic hinge around the circumference between the fracture surfaces. The number of points of failure around the circumference corresponds to a critical unstable mode of vibration.

\section{ACKNOWLEDGEMENTS}

The work presented in this paper was sponsored by AWE and EPSRC (DTA) and is gratefully acknowledged.

\section{REFERENCES}

[1] Taylor, G.I., The fragmentation of tubular bombs. The Scientific Papers of Sir Geoffrey Ingram Taylor Volume 1, Cambridge University Press, 1958.

[2] Mott, N.F., Fragmentation of shell cases. Proceedings of the Royal Society of London Series A: Mathematical and Physical Sciences, 189, pp. 300-308, 1947. doi:http:// dx.doi.org/10.1098/rspa.1947.0042 
[3] Rinehart, J.S. \& Pearson, J., Conical surfaces of fracture produced by asymmetrical impulsive loading. Journal of Applied Physics, 23(6), pp. 685-687, 1952. doi: http:// dx.doi.org/10.1063/1.1702279

[4] Singh, S., Corner fractures in explosively loaded thin-walled steel cylinders. British Journal of Applied Physics, 7(12), pp. 453-454, 1956. doi: http://dx.doi.org/10.1088/0508$\underline{3443 / 7 / 12 / 408}$

[5] Hoggatt, C.R. \& Recht, R.F., Fracture behaviour of tubular bombs. Journal of Applied Physics, 39(3), pp. 1856-1862, 1968. doi: http://dx.doi.org/10.1063/1.1656442

[6] Rushton, N.J., Schleyer, G.K., Clayton, A.M. \& Thompson, S., Internal explosive loading of steel pipes. Thin-Walled Structures 46, pp. 870-877, 2008. doi: http://dx.doi. org/10.1016/j.tws.2008.01.027

[7] Kobylkin, I.F., Calculation of the critical detonation diameter of explosive charges using data on their shockwave initiation. Combustion, Explosion and Shock Waves, 42(2), pp. 223-226, 2006. doi: http://dx.doi.org/10.1007/s10573-006-0042-6

[8] Cooper, P.W., Explosives Engineering, Wiley-VCH: New York, 1996.

[9] Grady, D.E. \& Kipp, M.E., Mechanisms of dynamic fragmentation: factors governing fragment size. Mechanics of Materials, 4, pp. 311-320, 1985. doi: http://dx.doi. org/10.1016/0167-6636(85)90028-6

[10] Goodier, J.N. \& McIvor, I.K., The elastic cylindrical shell under nearly uniform radial impulse. Journal of Applied Mechanics, 31, pp. 259-266, 1964. doi: http://dx.doi. org/10.1115/1.3629595

[11] Karpp, R.R., Duffey, T.A. \& Neal, T.R., Response of containment vessels to explosive blast loading. Pressure Vessel and Piping Division (ASME) Conference, 82-PVP-64, Orlando, Florida, June 1982. 